\title{
Traumatismo craneoencefálico secundario a heridas por arma de fuego atípica. Presentación de caso
}

Traumatic brain injury secundary to atipical gunshot-wound. A case report

\author{
Juan Carlos Morales Valdés. ${ }^{1}$, Diana Rosa Bayona Santana. ${ }^{2}$, Lilibeth Villalonga \\ Costa. ${ }^{3}$, Yusely Perdomo Pulido. ${ }^{4} \&$ Joel Socorro Izquierdo. ${ }^{5}$
}

\section{DOI: https://doi.org/10.33262/anatomiadigital.v4i3.1759}

\begin{abstract}
Introduction: Traumatic brain injury secondary to gunshot wounds (HPAF) is a critical situation. The projectile of a firearm can penetrate the body through any region, causing lesions of varied morphology due to multiple factors. The place of entry of the projectile (orifice) may have small diameters, which escapes the observation of the attending physician, in these circumstances radiological techniques are essential as a diagnosis and a preliminary step to the

\section{Resumen}

Introducción: $\quad \mathrm{El}$ traumatismo craneoencefálico (TCE) secundario a heridas por arma de fuego (HPAF) es una situación crítica. El proyectil de un arma de fuego puede penetrar al organismo por cualquier región originando lesiones de morfología variada por múltiples factores. El lugar de entrada del proyectil (orificio) puede tener diámetros pequeños, que escapa a la observación del médico de asistencia, en estas circunstancias se hacen indispensables

1 Hospital Universitario Comandante Faustino Pérez Hernández, jcmorales@infomed.sld.cu https://orcid.org/0000-0002-2756-3634

2 Hospital Universitario Comandante Faustino Pérez Hernández, dianar.mtz@infomed.sld.cu (DD https://orcid.org/0000-0001-6652-3558

3 Hospital Universitario Comandante Faustino Pérez Hernández, lilibeth.mtz@infomed.sld.cu iD https://orcid.org/0000-0002-4007-8908

${ }^{4}$ Policlínico Carlos Verdugo Martínez, yuselyp.mtz@infomed.sld.cu iD https://orcid.org/0000-0003-0574324

${ }^{5}$ Hospital Universitario Comandante Faustino Pérez Hernández, joels.mtz@infomed.sld.cu https://orcid.org/0000-0003-0743-850X
\end{abstract}


judicial autopsy. Methodology: A review and analysis of the bibliography on Traumatic brain injury caused by firearms was carried out, it was possible to identify orifices or entry wounds through natural orifices or other regions of the body, not because of unusual locations as in this case, being necessary to carry out tomographic studies to locate the projectile, which allowed to have it as a reference in future cases. Conclusions: The victim presented injuries, with severe head trauma. The tomography showed the presence of an intracerebral foreign body, similar to a projectile. The wound consisted of an atypical entrance orifice, in an unusual anatomical region, and a trajectory. At necropsy, the bullet was removed. The ballistics analysis revealed that it was a 22 caliber, of non-industrial manufacture, used in an atypical and improvised weapon. The intracranial injuries were severe, which by a direct mechanism caused the death of the injured person. The case was closed as a homicide.

Keywords: Improvised firearms, unusual wound or hole, unusual anatomical region, traumatic brain injury. las técnicas radiológicas como diagnóstico y paso previo a la necropsia judicial. Metodología: Se realizó una revisión y anàlisis de la bibliografia sobre los traumatismos craneoencefálicos por disparo de arma de fuego, se pudo identificar orificios o heridas de entrada por orificios naturales $\mathrm{u}$ otras regiones del cuerpo, no así por localizaciones poco comunes como en este caso, siendo preciso realizar estudiós tomográficos para localizar el proyectil, lo que permitió tenerlo como referencia en pròximos casos. Conclusiones: La víctima presentó lesiones, con severo traumatismo craneoencefálico. $\quad \mathrm{La}$ tomografía mostró la presencia de un cuerpo extraño intracerebral, semejante a un proyectil. La herida estaba formada por un orificio de entrada atípico, en región anatómica, poco común y un trayecto. En la necropsia realizada se extrajo la bala. En el análisis de balística resulto ser un calibre 22 , de fabricación no industrial, usado en un arma atípica e improvisada. Las lesiones intracraneales fueron severas y por un mecanismo directo ocasionaron la muerte del lesionado. El caso se cerró como un homicidio.

Palabras claves: Armas de fuego improvisada, herida u orificio atípico, región anatómica poco común, traumatismo craneoencefálico.

\section{Introducción.}

La violencia es una preocupación en la vida de las personas en todo el mundo, y afecta a todos de alguna u otra manera. Para muchos, poder estar a salvo se basa en mantener las puertas y ventanas cerradas, y evadir los lugares peligrosos. Sin embargo para otros no hay escapatoria, ya que la amenaza de la violencia se encuentra detrás de sus puertas, oculta a los ojos de los demás (Salas Castillo, D. D. R. ,2019). 
El trauma craneoencefálico (TCE) es una forma en la que la violencia se puede mostrar, es el intercambio de energía dentro de la bóveda craneana por un agente externo que resulta en alteraciones anatómicas o funcionales; se pueden generar lesiones tanto primarias, en cuanto al daño mecánico, como secundarias, de acuerdo al daño celular posterior (Alexiou, G. A., Sfakianos, G., \& Prodromou, N. ,2011).

Los TCE son la principal causa de muerte e incapacidad en individuos menores de 45 años en los Estados Unidos y la primera causa de muerte en los jóvenes de entre 1 a 19 años, fallecen entre 30000 y 50000 personas de manera secundaria a heridas por proyectil de arma de fuego, ocurren alrededor de 2,5 millones de casos anuales, la tasa de hospitalización es de un $11 \%$, con una mortalidad del $2 \%$, el $43,3 \%$ de los pacientes presentan algún grado de discapacidad al año del egreso, aproximadamente 150000 heridas por proyectil de arma de fuego se reportan por año (Corrigan J. D. ,2010 y Robles, C. ,2013 y Robles, C. ,2013).

América Latina es la región del mundo donde con mayor frecuencia son utilizadas las armas de fuego para realizar homicidios (66\%), en África y Asia (28\%), en Europa (13 $\%$ ); en Oceanía (10 \%) y en México, en la última década las armas de fuego han sido utilizadas en más de la mitad de los homicidios según los reportes publicados (Arriaga $\mathrm{P}$, Roldán Álvarez M. ,2016).

Gran parte de los traumas craneoencefálicos se asocian a heridas por arma de fuego en la región del cráneo, en el macizo frontal, por ser esta zona anatómica muy vascularizada y tener escasa musculatura, se caracterizan por favorecer a las hemorragias intensas, la localización de los órganos de los sentidos en esta región le da una especial sensibilidad al poderse afectar las vías respiratorias, los ojos u otras estructuras cercanas (Cañadas, E. V. ,2018 y Palacios Vivar, D. E., Miranda Villasana, J. E., \& Calderón Lumbreras, A. S. ,2017).

El estudio de las lesiones por arma de fuego en el cráneo forma parte de uno de los temas clásicos, constantes y fundamentales en todos los tratados de Medicina Legal. Los médicos deben conocer sus manifestaciones para su correcta interpretación desde la óptica quirúrgica y forense. Su producción exige siempre la investigación judicial por lo que la participación especializada desde la Medicina Legal es imprescindible para la resolución del caso (Canseco Cavazos, J., Palacios-Zertuche, J., Reyna-Sepúlveda, F., Álvarez-Villalobos, N., Alatorre-López, L., Muñoz-Maldonado, G. ,2017).

El TCE se puede clasificar de acuerdo a los mecanismos de traumatismo en penetrante, compuesto por heridas por arma corto-punzante, heridas por arma de fuego y cerrado, debido a caídas y accidentes de tránsito (Alexiou, G. A., Sfakianos, G., \& Prodromou, N. ,2011). La severidad del TCE se determina, a partir de la escala de coma de Glasgow (GCS) ajustada según la edad de los pacientes (Borgialli D. A. ,2016). Según Calabuig 2018 a la frecuencia y la gravedad de las lesiones craneoencefálicas se les confiere gran importancia, alrededor de una cuarta parte de las muertes violentas se deben a traumatismos de la cabeza, las lesiones que resultan pueden afectar a las partes blandas, al esqueleto craneofacial o a su contenido visceral. 
El Arma de Fuego, es un instrumento de dimensiones y formas variadas destinado a lanzar violentamente proyectiles, hacen uso de la fuerza expansiva de los gases que se desprenden al inflamarse de forma instantánea la sustancia explosiva en un espacio confinado. Conservan el nombre genérico de arma de fuego porque en los modelos primitivos los disparos iban acompañados de la salida de una llamarada por la boca del arma (Cañadas, E. V. ,2018).

Según su alcance y en relación con la longitud del cañón: las armas de fuego son cortas, (intervienen con mayor frecuencia en los asuntos criminales), entre ellas: pistolas ordinarias, revólveres, pistolas automáticas y pistolas ametralladoras o largas, (para la caza y con fines de guerra), dentro de estas se encuentran las escopetas de caza, fusiles, carabinas, fusiles ametralladoras, subfusiles y metralletas. Según la carga que disparan, pueden ser armas de proyectiles múltiples o de proyectil único (Cañadas, E. V. ,2018). Según la constitución de las armas de fuego, se clasifican en armas típicas, todas las armas comerciales fabricadas en serie por las distintas marcas o armas atípicas, siendo estas irregulares e improvisadas (Cañadas, E. V. ,2018). Refiere además el autor la existencia de nuevas innovaciones para el uso de armas con fines bélicos, se ha desarrollado un nuevo tipo de fusil de asalto, dotados de proyectiles de pequeño calibre y alta velocidad (Calabuig 2018).

Los proyectiles pueden ser puro de plomo o recubiertos con elementos como el cobre, bronce, zinc, los cuales conservan su forma primaria tras el impacto. En cuanto a la pólvora, en caso de la negra esta puede ser ordinaria, fuerte y extra-fuerte (dependiendo del número de granos de azufre, carbón y salitre) (Peña Coto, C. ,2013).

Para una mejor comprensión sobre el daño que ocasionan los proyectiles de las armas de fuego, nos adentraremos en las consideraciones que realiza la balística: La balística médico-forense es la parte de la balística externa que estudia el movimiento de la bala después de su impacto o penetración en el blanco corporal hasta el fin de su trayectoria o de su salida del cuerpo. La balística interior: es la que se ocupa del estudio de los fenómenos que ocurren en el interior del arma hasta que el proyectil sale por la boca del cañón, la balística exterior: estudia los fenómenos que ocurren en el proyectil desde el momento en que sale del arma hasta que alcanza su objetivo y la balística terminal: estudia la penetración del proyectil en cuerpos sólidos (Peña Coto, C. ,2013).

El grado de daño ocasionado tiene relación relativa a la energía ocasionada por la masa y velocidad de impacto del proyectil, la cual se representa con una fórmula y se le conoce como energía cinética (EC) siendo la velocidad más importante que la masa $(\mathrm{EC}=1 / 2 \mathrm{Masa}$ x Velocidad) (Hernández Téllez, I. E., Montelongo Mercado, E. A., Arreola Bastidas, J. J., Larrinúa Betancourt, E. \& Aguilar Merlo, A. ,2016).

La física cataloga el movimiento del proyectil en tres partes, interior (dentro cañón), exterior (desde su salida hasta su impacto), terminal (penetración en objetos sólidos). Presentando estos diferentes patrones de desplazamiento: precesión, caída y balanceo, 
cabeceo, sedal y nutación. No hay un acuerdo universal para clasificarlos por velocidad: la literatura de norteamericana refiere como alta velocidad entre 610-914 m/s, el Reino Unido toma mayor a $335 \mathrm{~m} / \mathrm{s}$ como alta velocidad se clasifican en menor a $330 \mathrm{~m} / \mathrm{s}$, media entre 330-600 m/s, y mayor a 600 m/s (Hernández Téllez, I. E., Montelongo Mercado, E. A., Arreola Bastidas, J. J., Larrinúa Betancourt, E. \& Aguilar Merlo, A. ,2016).

$\begin{array}{ccl}\text { Clasificación de Sherman y Parrish } & \\ \text { Baja velocidad } & <330 \mathrm{~m} / \mathrm{s} & \text { Deportivas recreativas } \\ \text { Mediana velocidad } & 330-660 \mathrm{~m} / \mathrm{s} & \text { Armas cortas, auto/semiautomáticas } \\ \text { Alta velocidad } & >600 \mathrm{~m} / \mathrm{s} & \text { Uso militar }\end{array}$

Respecto a la velocidad del proyectil describe que los fusiles nuevos de asalto, que expulsan proyectiles de pequeño calibre y alta velocidad, alcanzan los $1.000 \mathrm{~m} / \mathrm{s}$, en contraste con la velocidad de los proyectiles de revólver o pistola (de 200 a $300 \mathrm{~m} / \mathrm{s}$ ), o los de los fusiles ordinarios (de 600 a $700 \mathrm{~m} / \mathrm{s}$ ). El proyectil de alta velocidad genera una mayor energía cinética comparado con los de baja velocidad, pero asumir que ocasionan un mayor daño es una percepción equivocada. (Cañadas, E. V. ,2018).

El nivel de injuria va a depender de múltiples factores: energía cinética, capacidad de deformación, fragmentación de la bala y la resistencia a la deformación que presente el tejido alcanzado. Es de relevancia no sólo la velocidad sino también la zona de impacto, la energía cinética, características de la bala, y el trayecto de penetración para definir el daño, dar un pronóstico y un plan de tratamiento. Al momento de ingresar el proyectil en el organismo crea una cavidad cercana al diámetro del proyectil, una cavidad temporal ocasionada por expansión de la energía cinética de manera lateral, y una onda de estrés hacia delante. Teniendo en cuenta que la velocidad mínima para perforar la piel es de 50 $\mathrm{m} / \mathrm{s}$, y para afectar el hueso $60 \mathrm{~m} / \mathrm{s}$.

Existen múltiples clasificaciones en función del patrón de entrada y salida del proyectil: la primera distingue tres tipos, lesiones penetrantes (sólo orificio de entrada); lesiones perforantes (orificio de entrada y salida), la segunda por armas de pequeño calibre y larga distancia: lesiones avulsivas (provocadas por armas a una distancia muy corta con balas deformantes que generan gran pérdida de sustancia), la tercera describe cuatro trayectorias comunes del proyectil a nivel facial y la cuarta las clasifica en lesiones de bajo y alto grado de energía, en relación a la pérdida de sustancia y a las lesiones provocadas en la cara. (Teme Lichi, C., Gómez, N., Matsumura Kichiro, Cabral, G., \& Jara, J. ,2020).

Los elementos que integran al disparo son: la pólvora, el taco y el proyectil. La pólvora constituye la mezcla explosiva que comunica su fuerza de propulsión al proyectil. Pero también puede obrar sobre el blanco por sus productos de combustión: gases de explosión, llama, grano de pólvora no quemado y negro de humo (Cañadas, E. V. ,2018).

Se conocen como heridas por arma de fuego aquellos efectos lesivos que producen sobre el organismo los disparos realizados con armas cargadas de diversos tipos de pólvora u otros explosivos, son el conjunto de alteraciones producidas en el organismo por el efecto 
de los elementos que integran el disparo en las armas de fuego. Desde el punto de vista médico legal, las heridas por arma de fuego se clasifican como contusas (Alvarado, E. V. ,2014). Pueden provocar daños muy distintos dependiendo del intercambio de energía entre el cuerpo y la bala, además todas ellas tienen un orificio de entrada, una trayectoria y puede tener o no un orificio de salida que depende de la salida o no del proyectil del cuerpo (Gil Martín, P. ,2018).

Respecto a la morfología de las heridas por arma de fuego, dada sus sensibles diferencias, tenemos las heridas producidas por proyectil único (bala) y las causadas por proyectiles múltiples. Describiremos la que se relaciona en este caso, que sería la producida por proyectil único. De manera esquemática, hay que considerar en estas heridas un orificio o herida de entrada, un trayecto y un orificio o herida de salida, el cual puede o no estar (Cañadas, E. V. ,2018).

La forma del orificio es generalmente, redondeada u oval, en el vivo es excepcional que sea totalmente circular, pues siempre hay una deformación conforme a la dirección de las fibras de la región (como en las heridas punzantes). En las heridas por disparos a gran distancia, el orificio adopta la forma de un ojal o hendidura lineal que recuerda las heridas por instrumentos punzantes o cortopunzantes. En Las heridas por disparos a corta distancia la forma de la herida presenta un aspecto desgarrado, como de estrella, debido a la acción de los gases, que se difunden con violencia bajo la piel (Alvarado, E. V. ,2014).

Los tatuajes son las formaciones resultantes del disparo que se dibujan alrededor del orificio de entrada y suministran importantes indicaciones diagnósticas médico legal, tienen dos componentes: la cintilla de contusión y el taraceado o tatuaje propiamente dicho. En algunas ocasiones, el orificio de entrada de las heridas por arma de fuego difiere de la descripción anterior, dando lugar a las heridas atípicas. Cuando el proyectil entra por orificios o cavidades orgánicas, se habla de orificio de entrada natural (boca, fosas nasales, conducto auditivo externo, vagina y ano (Alvarado, E. V. ,2014).

El trayecto de las heridas por arma de fuego corresponde al recorrido de la bala en los tejidos, puede ser único o múltiple, si la bala se fragmenta durante su paso por los tejidos. Por su dirección se distinguen los trayectos en rectilíneos, que siguen la dirección del disparo, y los trayectos con desviación, por choques en huesos, sobre todo, que comunican a los proyectiles hacia direcciones sorprendentes, indescriptibles (Alvarado, E. V. ,2014).

Si el proyectil no ha salido al exterior, se encuentra en la extremidad del trayecto cerrado, en medio de un foco hemorrágico. Sin embargo, la búsqueda del proyectil es a menudo muy dificultosa, ya que puede ocupar lugares inverosímiles por mecanismos no siempre bien explicados. Para facilitar la localización de la bala se hace a veces necesario el concurso de la imagenología. En ocasiones es aconsejable pasar los coágulos sanguíneos encontrados en las cavidades (abdominal, torácica, etc.) por un tamiz, con el fin de encontrar los proyectiles que a veces quedan englobados en ellos (Peña Coto, C. ,2013).

Se describen además las migraciones, que no es más que el traslado pasivo del proyectil por medio de la corriente sanguínea, cuando este penetra en un vaso. Por otra parte, la 
dirección del trayecto en el cuerpo depende de la posición en la que se encontraba la víctima. El orificio de salida es inconstante por definición, no existiendo cuando la bala permanece en los tejidos, variando su forma y su tamaño (Alvarado, E. V. ,2014).

Las heridas por arma de fuego son de naturaleza contusa y físico químicamente ocasionadas por la bala o proyectil disparado por un arma de fuego, y por los elementos concurrentes, más los elementos neoformados con ocasión del disparo. Las lesiones intracraneales secundarias a heridas por proyectil de arma de fuego son generalmente múltiples y variadas, pueden ocasionar compromiso óseo, parenquimatoso e incluso vascular (Peña Coto, C. ,2013).

Las armas de fuego, dependiendo del tipo de proyectil y la distancia del disparo, pueden producir: Fracturas incompletas (fisuras que se irradian y que se interrumpen en las suturas o en fisuras o fracturas producidas por disparos anteriores). Orificios, bien dibujados, con fisuras o microfracturas, que se irradian perpendiculares al círculo del orificio. Orificios irregulares, de gran tamaño, cuando se suma el efecto explosivo («heridas a bocajarro»); en ocasiones, la explosión puede producir fracturas indirectas. (Arriaga P., Roldán Álvarez M. ,2016).

Las complicaciones más frecuentes ante este tipo de lesión que conllevan al deceso son: Las fracturas que se producen al ocurrir el encuentro entre el proyectil y el segmento óseo, y su trayecto a través del mismo. La alteración vascular por rupturas de vasos sanguíneos cerebrales, que dan pasó a la formación de hematomas y hemorragias intracraneales y la lesión de los centros nerviosos superiores. (Canseco Cavazos, J., Palacios-Zertuche, J., Reyna-Sepúlveda, F., Álvarez-Villalobos, N., Alatorre-López, L., Muñoz-Maldonado, G. ,2017).

El caso que se presenta fue trabajado por el Servicio Provincial de Medicina Legal de la provincia de Matanzas, la autoridad competente (Instructor Policial) solicita que se realice la necropsia médico legal al cadáver de un individuo que encontrándose con vida ingresa en sala de emergencia con pérdida de la conciencia, múltiples estigmas de traumatismos y sangramiento activo por boca, fosas nasales y el ángulo interno de la región orbital derecha, falleciendo horas más tardes.

Entre los estudios que se le realizaron se encuentra una tomografía axial computarizada simple (TAC) de la región craneal, la cual reveló la presencia de un cuero extraño con un aumento de la densidad, alojado en el Encéfalo, en su hemisferio izquierdo a nivel parietal.

En la necropsia realizada se evidenció el orificio de entrada de una herida por arma de fuego, su trayecto, lesiones y el propio proyectil. La herida tenía una pequeña dimensión y una localización poco común, que pudo escapar a la observación del médico de asistencia, pero en este caso el hallazgo del cuerpo extraño observado en la tomografía, sugería por su densidad la posibilidad de un proyectil de arma de fuego. 


\section{Metodologia.}

Síntesis de los Hechos:

Se conoció a través de la información aportada por la autoridad actuante, que la víctima sostuvo una riña en la vía pública con otro ciudadano, quien lo agrede con un pedazo de acero o cabilla, causándole lesiones en la cabeza. Fue encontrado por los vecinos, cerca de la vivienda donde este reside, sobre el pavimento, inconsciente, con presencia de golpes en la región de la cara y sangramiento abundante, siendo trasladado a un centro hospitalario con peligro inminente para su vida, por presentar toma de conciencia, lesiones y sangramiento activo. Después de varias horas del accionar médico, el lesionado fallece.

Presentación del caso:

El caso que se presenta esta en relación con una denuncia radicada por la autoridad competente por el delito de homicidio al tratarse de la muerte de una persona por otra, donde la víctima fue agredida supuestamente con un objeto contundente, recibiendo varias lesiones, en la región cefálica y miembro superior derecho. El lesionado fue traslado inmediatamente al centro hospitalario, siendo valorado en sala de emergencia, lo que consta en su historia clínica, donde se describe que desde su llegada se continua brindando asistencia médica y es valorado por médicos de diferentes especialidades, dentro de ellas Neurocirugía por la toma de conciencia y la presencia de una escala de Glasgow de 3 sobre 15, lo que orientó a los galenos sobre la presencia de lesiones intracraneales. Indican y realizan Tomografía Axial Computarizada de cráneo simple $(T A C)$ donde se observa la presencia de neumoencéfalo, fractura de la pared medial de seno maxilar derecho, el cual se encontraba ocupado por contenido hemático, fractura en el etmoides, el cual está en similares condiciones, fractura de la pared medial de la órbita izquierda y fractura de la pared lateral del seno esfenoidal izquierdo, ocupado por contenido hemático y con ocupación parcial del derecho, además se constató la presencia de un cuerpo extraño intracerebral, alojado en el hemisferio izquierdo, a nivel de la región parietal de ese lado, próximo a la línea media, con una densidad de $3034 \mathrm{UH}$, que impresionaba elemento de composición metálica, similar a un proyectil de arma de fuego. Al no reportarse agresión con arma de fuego ni evidenciarse un orificio de entrada en tal región anatómica, surgió la duda ante tal elemento.

Se discute el caso en colectivo en presencia del médico legista (medicina legal), para exponer las lesiones visibles, su mecanismo de formación y posible agente vulnerante. Al examinarse la región orbital derecha, se evidencia un activo sangramiento en el ángulo interno, con presencia de una herida contusa de $1,1 \mathrm{~cm}$ de diámetro, sin otros signos.

Durante el reconocimiento corporal de lesiones se observaron contusiones simples y profundas en el miembro superior derecho, en el antebrazo. Al analizar y discutir el caso, teniendo como referencia los estudios radiológicos (TAC), que evidenciaban la presencia de traumatismo craneal, y el cuadro lesivo descrito, se esclarecía aún más que este individuo había sido agredido, con un objeto contundente, pero que a la vez era muy 
probable que hubiese sido agredido también con un arma de fuego, teniendo como referencia que la herida contusa descrita en el ángulo interno de la región orbital derecha, era el orificio de entrada del cuerpo extraño que se encontraba alojado intracraneal. Al ser valorado por los especialistas de Neurocirugía, estos comentan en la historia clínica, que el lesionado no tenía criterio quirúrgico. Evolucionando tórpidamente desde el punto de vista neurológico y hemodinámico, fallece en un breve tiempo.

Realizada la necropsia médico legal por los peritos actuantes, evidenciaron los siguientes signos de violencia en el examen del exterior del cadáver:

- Aumento de volumen de la región ocular derecha, con equimosis y hematoma periorbitario de esta región, con coloración violáceo oscuro.

- Quemosis del ojo derecho.

- Herida contusa, de 1,1 cm de longitud, en forma de un pequeño ojal, localizada en borde interno del párpado inferior del ojo derecho, próximo al lagrimal, con evidente sangrado a través de la misma.

- Aumento de volumen del antebrazo derecho, endurecido a la palpación, donde se observa la presencia de 4 excoriaciones lineales, dispuestas transversalmente al eje longitudinal del miembro superior, equidistantes y paralelas entre sí, de 7, 7,6, 8 y $10 \mathrm{~cm}$ de longitud, tomando como referencia la región distal del miembro, todas con costra húmeda y hemática. En la región correspondiente a la flexura del codo de ese mismo miembro, se evidencia hematoma de $6,7 \mathrm{~cm}$ en su diámetro mayor, con color violáceo oscuro.

Al examinar los orificios naturales solo se evidenció la presencia de contenido hemático en ambas fosas nasales, no existiendo alteraciones en su morfología.

En el examen del interior de las cavidades, como significativo se describe lo evidenciado a la apertura de la cavidad craneana:

- Infiltrado hemorrágico en el colgajo anterior del cuero cabelludo, galeal y subgaleal, en región parietal y frontal derecha, a nivel de la órbita y región nasal.

- Cerebro:

- Vasos meníngeos tortuosos e ingurgitados, aplanamiento de las circunvoluciones y estrechamiento de los surcos.

- Extravasación de sangre al espacio subaracnoideo, extensa, en el hemisferio izquierdo en su región parietal.

- En la base cerebral se observó dislaceración del tejido encefálico, en la misma se evidencia la presencia de un surco o canal en toda su extensión, dejando visualizar la presencia de un proyectil deformado en su interior.

Tallo encefálico: Tras su disección se observaron focos contusivos.

Huesos de la base craneal:

Fractura lineal, de $0,5 \mathrm{~cm}$ de longitud, en fosa anterior derecha, con infiltrado hemorrágico a su alrededor. 
- Por debajo de la lámina cribosa del etmoides se observa fractura lineal de $1,2 \mathrm{~cm}$ de longitud, que se extiende hasta la silla turca, con infiltración hemorrágica.

- Exploración del orificio de entrada: Una vez introducido el explorador metálico a través de la herida contusa descrita en el ángulo interno de la región orbital derecha, este atravesó la base craneal a través de un trayecto recto que pasa por la fosa anterior derecha.

- Una vez extraído el proyectil se evidencio que el mismo tiene características de estar deformado, construido de un material que nos recuerda al plomo, de 1,2 cm de longitud y $0,6 \mathrm{~cm}$ de ancho en su base.

- El estudio de balística realizado al proyectil en el laboratorio de criminalística, aportó que el elemento investigado era de Plomo, color gris, con un peso aproximado de 2.41 gramos, perteneciente a un proyectil de base cóncava y punta redonda, apreciándose su cuerpo con la cabeza deformado por un lateral, producto a un achatamiento, el cual pudo producirse producto al impacto contra una barrera de mayor dureza. No presenta estrías y campos, estuvo unido al casquillo por presión. Todas estas características unidas a las constructivas y a la comparación realizada con los patrones existentes en los registros criminalísticos de cartuchos, posibilitó establecer que: el proyectil formó parte de un cartucho Calibre 5,6mm (.22') de los diseñados para ser utilizados en diversas armas del tipo semiautomática y mecánica, además de armas atípicas.

Consideraciones médico legales

- Los peritos médicos legistas actuantes, consideran que teniendo en cuenta lo aportado por la autoridad actuante, lo descrito en la historia clínica que perteneciera al lesionado, los resultados del reconocimiento corporal de lesiones practicado y las evidencias obtenidas, tras realizar la necropsia judicial, nos encontramos ante una muerte violenta, la cual es dependiente de la agresión con arma de fuego, que conllevo a las lesiones craneoencefálicas descritas.

- El cuadro lesivo encontrado en la región craneoencefálica durante la realización de la necropsia médico legal, guarda relación con los hallazgos tomográficos evidenciados, no así con el antecedente aportado por la Instrucción Penal actuante en el inicio de la investigación.

- Orientar a la autoridad judicial, que la víctima no solamente había sido agredida con un objeto contundente, sino que también lo había sido con un arma de fuego, que tras el disparo del proyectil y el encuentro de este con el cuerpo (región cefálica), a nivel del ángulo interno de la región orbital derecha, había penetrado la bala, dejando la herida u orificio de entrada a la cavidad craneal de donde no salió, quedando alojado en el interior del tejido encefálico, en la región parietal izquierda, lo cual quedó evidenciado en el examen in situ realizado, no existiendo por tanto orificio de salida.

- El orificio de entrada en este caso tiene una localización poco común, con dimensiones y características que bien podrían confundirse con una herida contusa causada por otro objeto contuso e incluso escapar de la observación del médico 
de asistencia. En estas situaciones clínicas donde hay toma de conciencia si no se indican los estudios tomográficos correspondientes, no se diagnosticarían las lesiones causadas, ni la presencia del proyectil. Lo que sería importante para el médico legista, al poder tener localizado al proyectil antes de la realización de la necropsia judicial, en caso de fallecimiento del lesionado.

- Al analizar la morfología de las heridas por arma de fuego, a pesar de lo evidenciado en el estudio tomográfico realizado al lesionado, consideramos que en este hecho nos encontramos ante una producida por proyectil único, conformada por el orificio de entrada y su trayecto, al estar ausente el orificio de salida. El orifico de entrada en este caso no presenta las características descritas para los disparos a corta y larga distancia, dando lugar por lo tanto a una herida atípica, por lo que no podemos pronunciarnos respecto a la distancia del disparo.

- Durante la exploración del trayecto se evidenció que el mismo presenta desviación, al chocar con el plano óseo.

- Respecto a la dirección del disparo, solo tendremos en cuenta el trayecto del proyectil por ser esta una herida atípica, el cual sería de derecha a izquierda y de adelante hacia detrás, lo que justifica la herida contusa en el párpado inferior del ojo derecho, la fractura de la fosa anterior derecha del cráneo, la fractura por debajo de la lámina cribosa del etmoides, que se extiende hasta la silla turca y el resto de las lesiones ocasionadas por el trayecto del proyectil en el encéfalo.

- Después de analizado y evaluado el proyectil, se ilustro a la autoridad actuante que el mismo había sido disparado por un arma atípica, de constitución irregular, e improvisada.

- Que la deformación de la cabeza del proyectil producto a un achatamiento, puede estar en relación con el impacto que tuvo el mismo en su trayecto contra el plano óseo, lo que justifica las fracturas observadas en el estudio tomográfico y descritas en el cuadro lesional que se comprobó. Con los datos aportados y el estudio balístico realizado se concluyó que el proyectil formó parte de un cartucho Calibre 5,6mm (.22’) de los diseñados para ser utilizados en diversas armas del tipo semiautomática y mecánica, además de su uso en armas atípicas como lo fue categóricamente en este hecho.

- El traumatismo craneoencefálico que se originó secundariamente al disparo por el arma de fuego fue clasificado como severo, si tenemos en cuenta la escala de coma de Glasgow (GCS) que presentaba la víctima de 3/15 al momento de ser examinado por los galenos.

- El trauma craneoencefálico que presentó la víctima de acuerdo a los mecanismos de traumatismo se clasifica en penetrante.

- Las lesiones intracraneales descritas en la víctima, secundarias a la herida por el proyectil de arma de fuego fueron múltiples y variadas, teniendo compromiso óseo, parenquimatoso y vascular.

- Las complicaciones que presentaron estas lesiones conllevaron al lesionado a su deceso en breve tiempo, teniendo como mecanismo directo de muerte la lesión de los centros nerviosos superiores. 
- Los signos de violencia descritos en la región ocular derecha (equimosis y hematoma periorbitario), no son más que contusiones simples o de primer grado con integridad de la piel, originados por la ruptura de vasos sanguíneos y son secundarios a la herida por arma de fuego y sus complicaciones. Los descritos en el miembro superior derecho, a nivel de antebrazo son: excoriaciones, pertenecientes también al grupo de contusiones anteriores, pero con lesión cutánea, cuyo mecanismo de formación es por fricción o rozamiento y hematoma, causado por la ruptura de vasos sanguíneos.

- Pericialmente concluimos el caso declarando que se trata de una muerte violenta de etiología médico legal homicida tras la agresión de la víctima. El agente vulnerante usado por parte de él o de los agresores fue un objeto contundente, duro, romo, sin filo, ni aristas y un arma de fuego improvisada, atípica. Su disparo interesó la región craneoencefálica en una localización poco común, causando un traumatismo craneoencefálico. Presentaba otras lesiones en el antebrazo derecho (contusiones simples) pero sin repercusión a la gravedad de su estado. La causa directa de la muerte estuvo relacionada con la lesión de los centros nerviosos superiores, por la dislaceración encefálica y las contusiones de tallo encefálico que se presentaron al fracturarse la base del cráneo producto al disparo de proyectil único de arma de fuego, cuando la víctima fue agredida por tal agente vulnerante.

Fig. 1. Contusiones superficiales en miembro superior derecho.

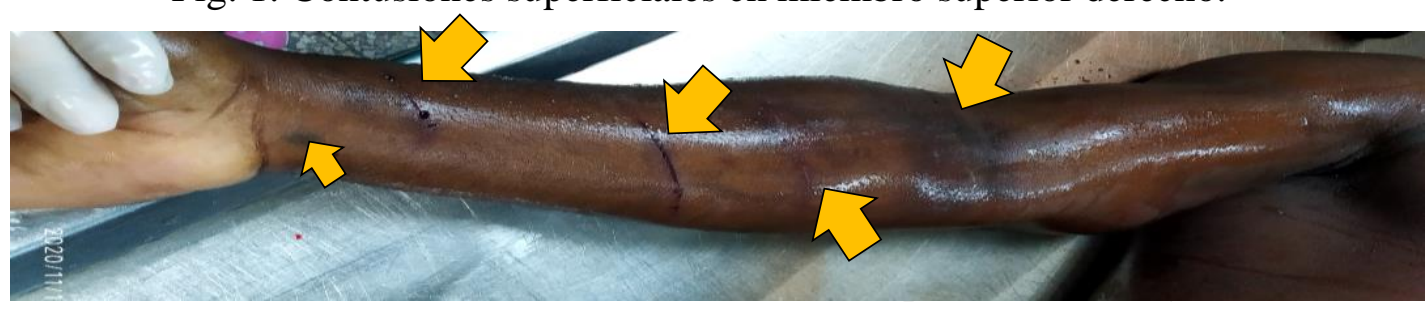

Fig. 2. Herida por arma de fuego con orificio de entrada atípico.

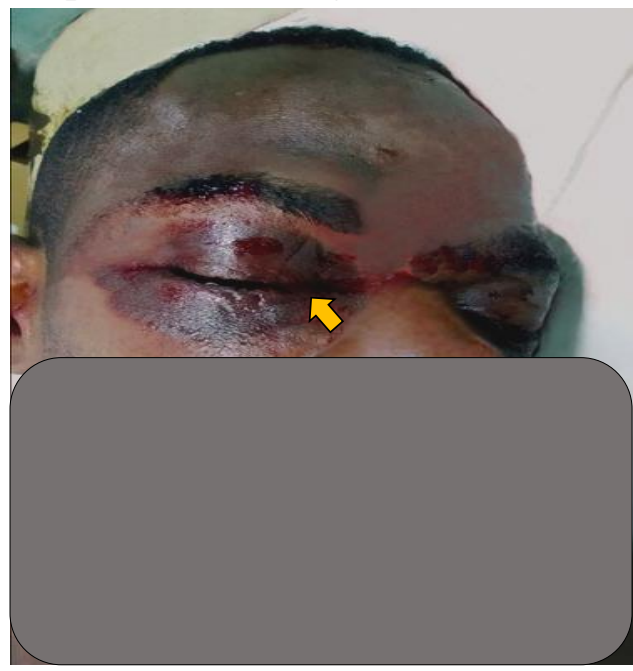

Fuente: Foto obtenida durante el acto de la necropsia Médico Legal. 
Fig. 3. Tomografía simple de cráneo

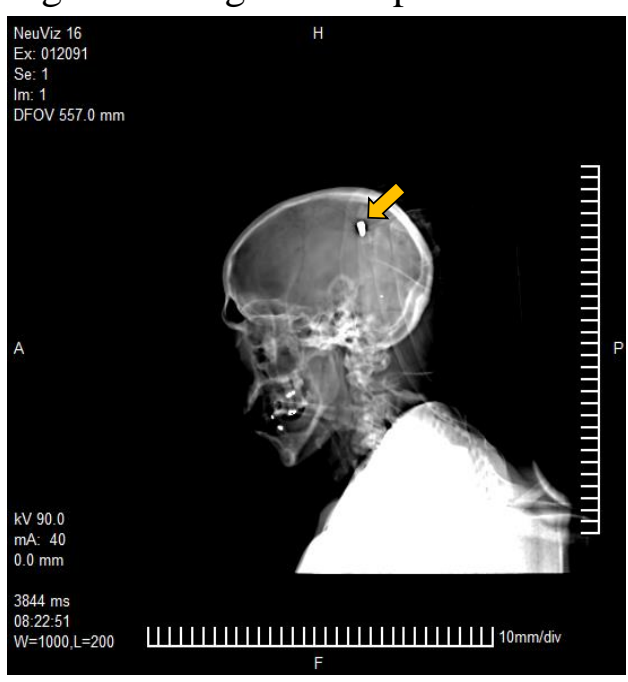

Fig. 4. Estudio tomográfico en 3D

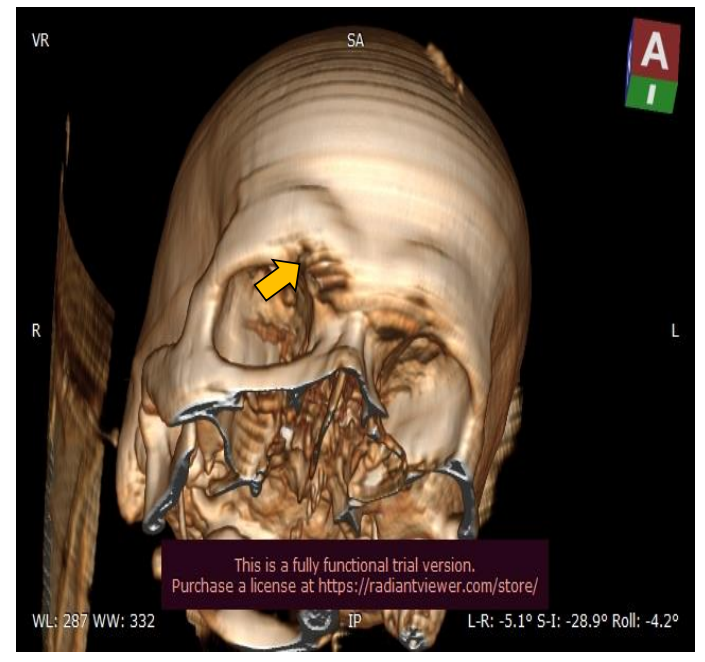

Fuente: Estudios imagenológicos realizados (Tomografía)

Fig. 5 Proyectil calibre 22 deformado

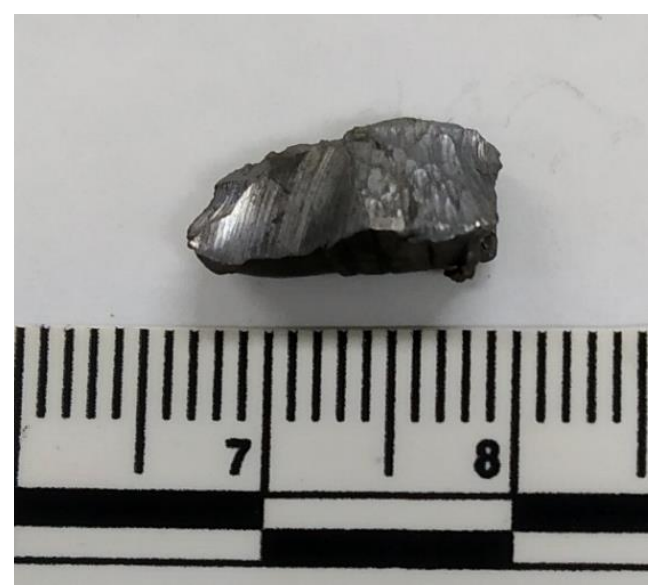

Fuente: Proyectil extraído en el acto de la necropsia Médico Legal

\section{Conclusiones.}

- La víctima presentó varias lesiones, con un severo traumatismo craneoencefálico. El estudio tomográfico mostró la presencia de un cuerpo extraño intracerebral, semejante a un proyectil. La herida solo tenía el orificio de entrada atípico, en una región anatómica poco común y un trayecto. Durante el acto de la necropsia médico legal se extrajo la bala. En el análisis de balística resulto ser un calibre 22, de fabricación no industrial, usado en un arma atípica e improvisada. Las lesiones intracraneales fueron severas y por un mecanismo directo ocasionaron la muerte del lesionado. El caso se cerró desde la perspectiva médico legal como un homicidio. 


\section{Referencias bibliográficas.}

Alexiou, G. A., Sfakianos, G., \& Prodromou, N. (2011). Pediatric head trauma. Journal of emergencies, trauma, and shock, 4(3), 403-408. https://doi.org/10.4103/09742700.83872

Alvarado, E. V. (2014). Libro de Medicina Legal. https://booksmedicos.org/medicinalegal-vargas-alvarado-4a-edicion/

Arriaga P., Roldán Álvarez M. (2016). Armas de fuego en México: Panorama en 2016, Publicaciones Casede. págs.: 157-167.4. https://www.casede.org/PublicacionesCasede/Atlas2016/Paulina_Arriaga_Maura_ Roldan.pdf

Borgialli, D. A., Mahajan, P., Hoyle, J. D., Jr, Powell, E. C., Nadel, F. M., Tunik, M. G., et al. (2016). Performance of the Pediatric Glasgow Coma Scale Score in the Evaluation of Children With Blunt Head Trauma. Academic emergency medicine: official journal of the Society for Academic Emergency Medicine, 23(8), 878-884. https://doi.org/10.1111/acem.13014

Canseco Cavazos, J., Palacios-Zertuche, J., Reyna-Sepúlveda, F., Álvarez-Villalobos, N., Alatorre-López, L., Muñoz-Maldonado, G. (2017). Epidemiología de las lesiones por proyectil de arma de fuego en el Hospital Universitario“'Dr. José Eleuterio González' de la Universidad Autónoma de Nuevo León Cirugía y Cirujanos, vol. 85, núm. 1, enero-febrero, pp. 41-48. Disponible en: http://www.redalyc.org/articulo.oa?id=66249769007

Cañadas, E. V. (2018). Gisbert Calabuig. Medicina Legal Y Toxicológica. Elsevier. https://www.elsevier.com/books/gisbert-calabuig-medicina-legal-ytoxicologica/villanueva-canadas/978-84-9113-096-3

Corrigan, J. D., Selassie, A. W., \& Orman, J. A. (2010). The epidemiology of traumatic brain injury. The Journal of head trauma rehabilitation, 25(2), 72-80. https://doi.org/10.1097/HTR.0b013e3181ccc8b4

Gil Martín, P. 2018. Manejo inicial del paciente con herida por arma de fuego. Disponible en: https://gredos.usal.es/handle/10366/137597

Hernández Téllez, I. E., Montelongo Mercado, E. A., Arreola Bastidas, J. J., Larrinúa Betancourt, E. \& Aguilar Merlo, A. (2016). Spine gunshot wounds at the Central Military Hospital in Mexico. Coluna/Columna, 15(2), 134-139. https://doi.org/10.1590/S1808-185120161502157642

Palacios Vivar, D. E., Miranda Villasana, J. E., \& Calderón Lumbreras, A. S (2017). Herida facial por proyectil de arma de fuego: revisión de literatura y estudio clínico de tres casos. Revista odontológica mexicana, 21(2), 127-134. https://doi.org/10.1016/j.rodmex.2017.05.009 
Peña Coto, C. (2013). Manejo de las heridas por proyectil disparado por arma de fuego en la sección de patología forense del departamento medicina legal del Poder Judicial, Costa Rica. Medicina Legal de Costa Rica, 30(2), 113-121. Retrieved March 15, 2021, from http://www.scielo.sa.cr/scielo.php?script=sci_arttext\&pid=S140900152013000200013\&lng=en\&tlng=es.

Pinilla, G., Castro, I. \& Amaya, C. (2018). Trauma craneoencefálico por proyectil de arma de fuego: revisión temática. Revista Criminalidad, 60 (2): 107-126. http://www.scielo.org.co/pdf/crim/v60n2/1794-3108-crim-60-02-00107.pdf

Pradere Pensado, J. C., García Gómez, A., Padrón Valdés, F. \& Coca Machado, J. L. (2016). Paciente con lesiones por balística terminal. Revista Cubana de Cirugía, 55(1) Recuperado en 15 de marzo de 2021, de http://scielo.sld.cu/scielo.php?script=sci_arttext\&pid=S0034-

$74932016000100008 \& \operatorname{lng}=$ es\&tlng=es.

Robles, C. (2013). Historia de las armas de fuego (en línea). Estados Unidos, Scribd. Consultado 24 feb. 2018. Disponible en https://es.scribd.com/doc/138364377/Historia-de-Las-Armas-de-Fuego.pdf

Teme Lichi, C., Gómez, N., Matsumura Kichiro, Cabral, G., \& Jara, J. (2020). Traumatismo cervical por arma de fuego en pediatría: apropósito de un caso. Pediatría (Asunción), 47(1), 39-43. Epub 00 de abril de 2020.https://dx.doi.org/10.31698/ped.47012020007

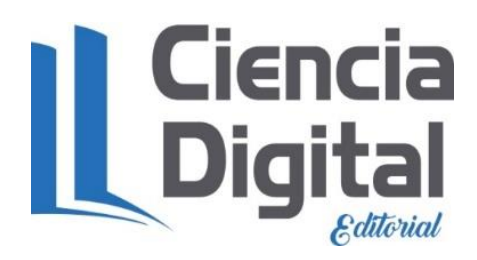




\section{PARA CITAR EL ARTÍCULO INDEXADO.}

Morales Valdés, J. C., Bayona Santana, D. R., Villalonga Costa, L., Perdomo Pulido, Y., \& Socorro Izquierdo, J. (2021). Traumatismo craneoencefálico secundario a heridas por arma de fuego atípica. Presentación de caso. Anatomía Digital, 4(3), 102-117. https://doi.org/10.33262/anatomiadigital.v4i3.1759

\section{Ciencia \\ LDigital}

El artículo que se publica es de exclusiva responsabilidad de los autores y no necesariamente reflejan el pensamiento de la Revista Anatomía Digital.

El artículo queda en propiedad de la revista y, por tanto, su publicación parcial y/o total en otro medio tiene que ser autorizado por el director de la Revista Anatomía Digital.
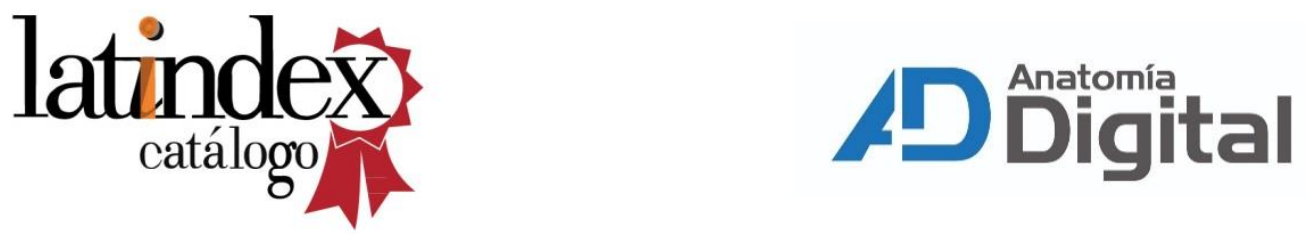